\title{
Application of Adaptive PSO and Adaptive Fuzzy Logic Controllers to Speed Control PMSM Motor Servo Systems
}

\author{
Le Dinh Hieu ${ }^{1,2}$, Temkin Igor Olegovich ${ }^{1}$ \\ ${ }^{1}$ Information Technologies - Automated Control Systems, National University of Science and Technology MISiS, Leninsky Prospekt 4, \\ 119049, Moscow, Russia. \\ ${ }^{2}$ Electrical-Electronics Faculty, Hue Industrial College, Hue City, 70 Nguyen Hue Street, 530000, Vietnam.
}

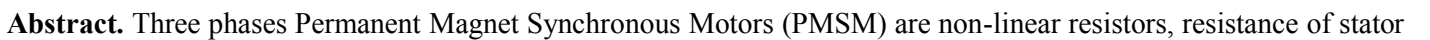

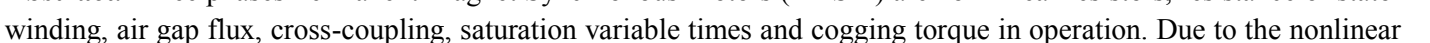

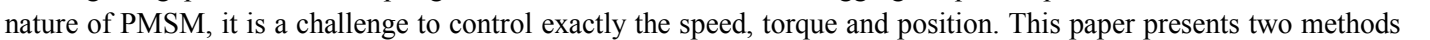

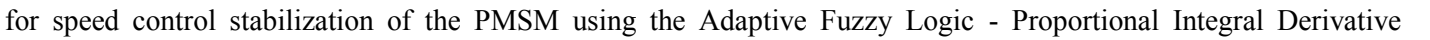

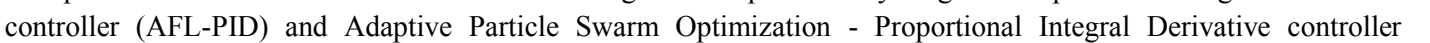

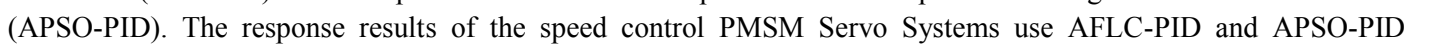

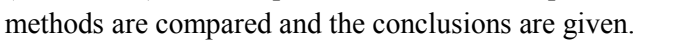

\section{Introduction}

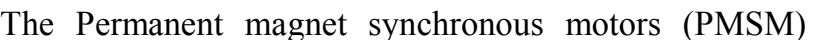

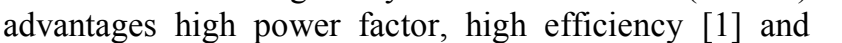

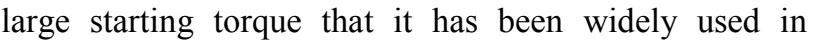

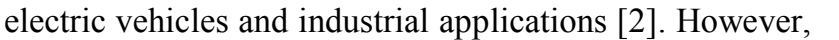

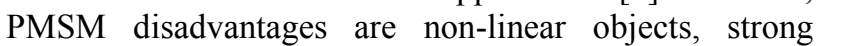

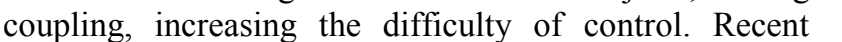

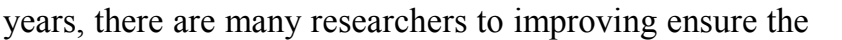

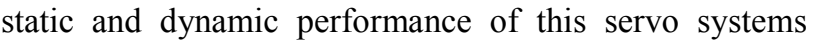

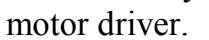

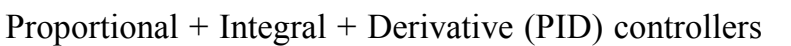

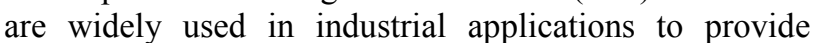

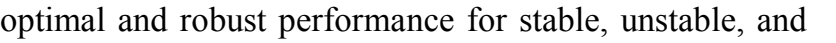

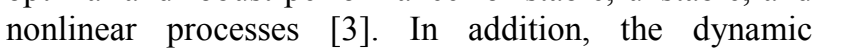

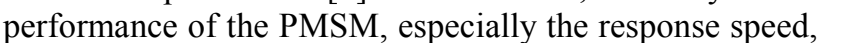

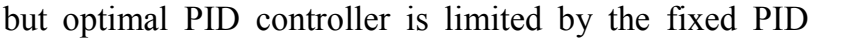

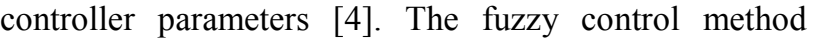

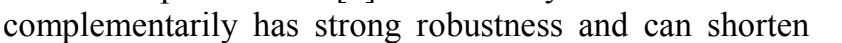

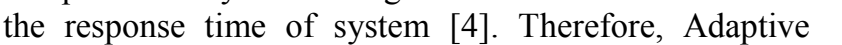

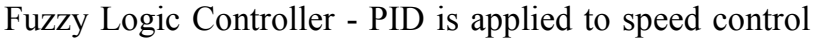

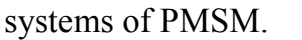

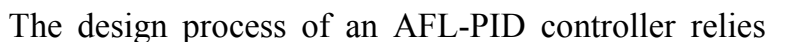

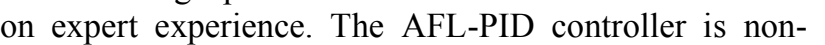

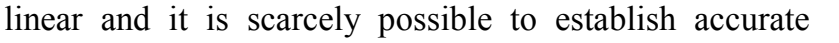

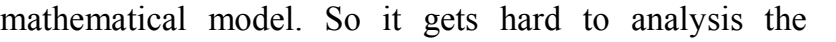

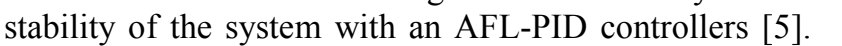

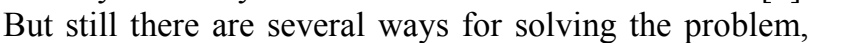

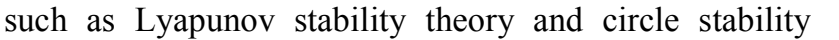

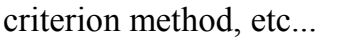

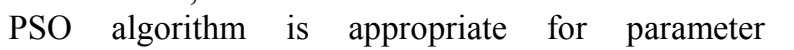

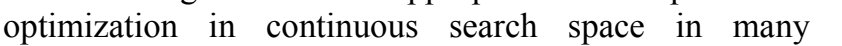

(C) The Authors, published by EDP Sciences. This is an open access article distributed under the terms of the Creative Commons Attribution License 4.0 (http://creativecommons.org/licenses/by/4.0/).

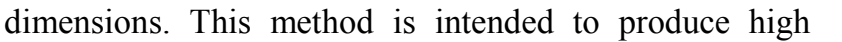

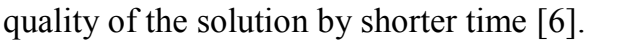

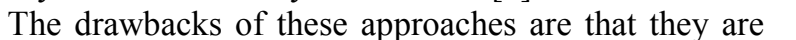

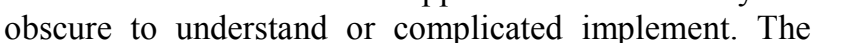

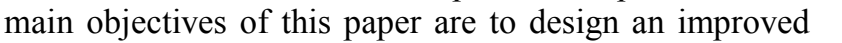

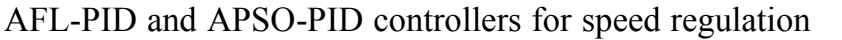

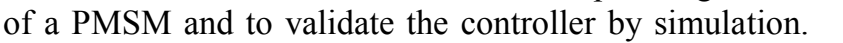

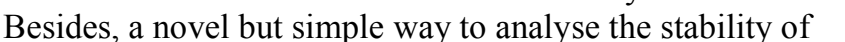

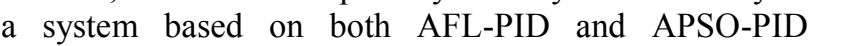

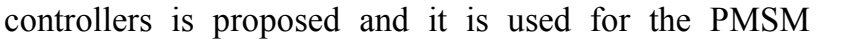

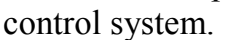

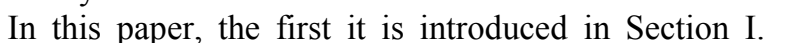

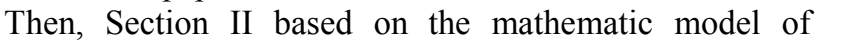

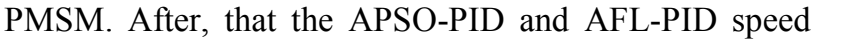

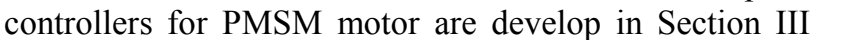

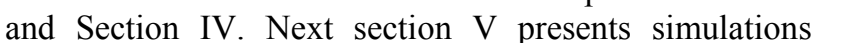

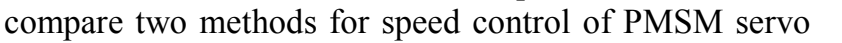

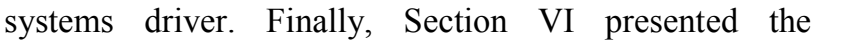

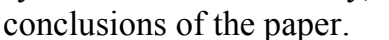

\section{Model mathematic of PMSM motor}

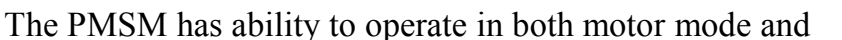

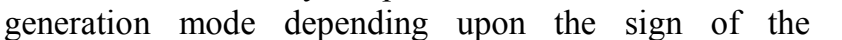

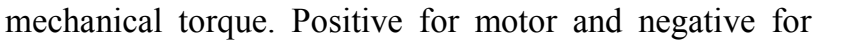

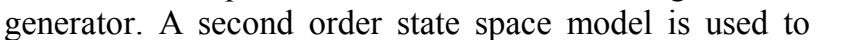

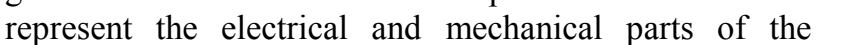

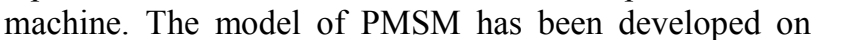

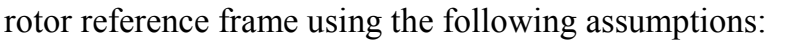

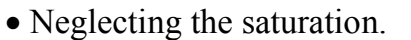

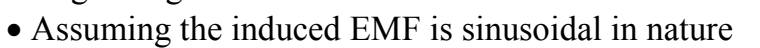

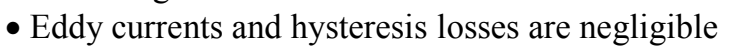

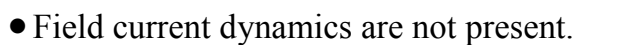

\section{$\square \quad \square \quad \square$}


The $\square \square \| \square$ of PMSM are given [7]:

$$
\begin{aligned}
& u_{d}=R i_{d}+\frac{d \lambda_{d}}{d t}-\omega_{e} \lambda_{q} \\
& u_{q}=R i_{q}+\frac{d \lambda_{q}}{d t}-\omega_{e} \lambda_{d} \\
& J \frac{d \omega_{m}}{d t}=T_{m}-T_{L}-B \omega_{m} \\
& T_{m}=\frac{\square}{\square} \square \lambda_{d} i_{q}-\lambda_{q} i_{d} \square
\end{aligned}
$$

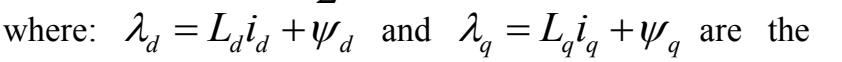

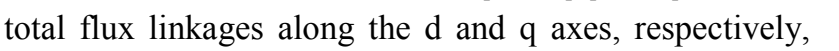

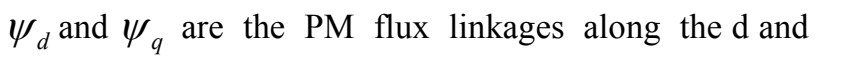

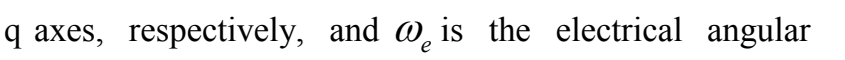

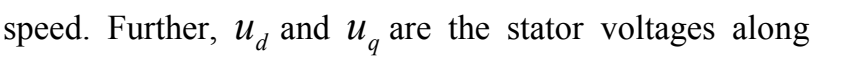

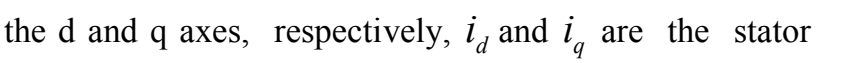

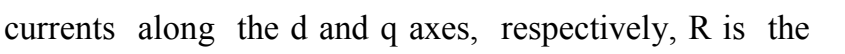

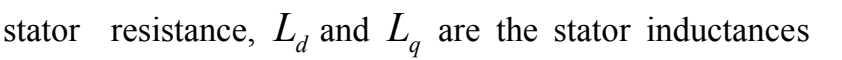

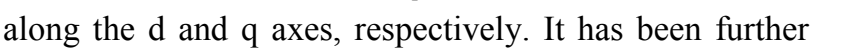

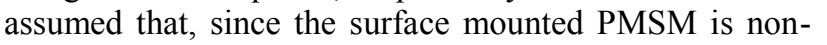

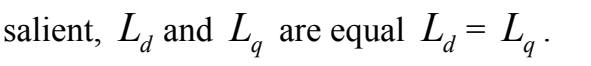

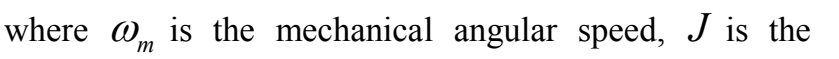

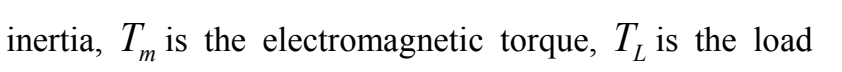

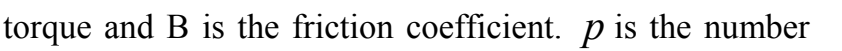

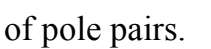

\section{Adaptive PSO-PID speed controller PMSM}

\subsection{PSO optimal the parameters PID}

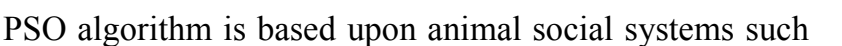

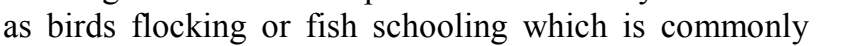

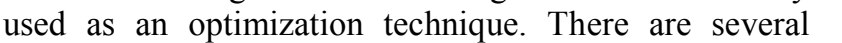

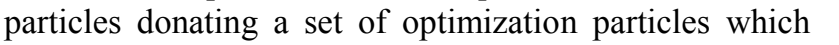

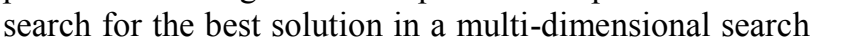
एण

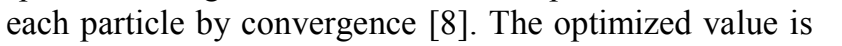

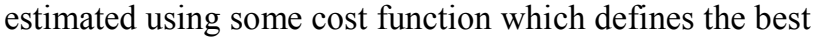

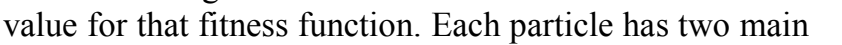

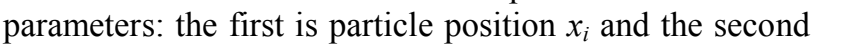

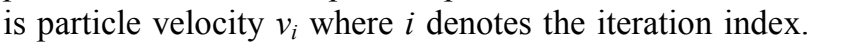

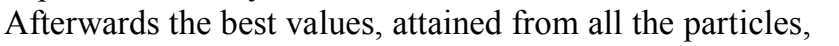

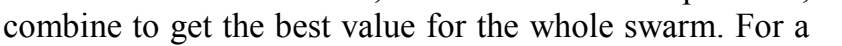

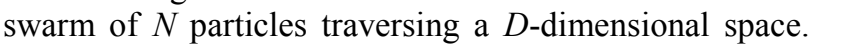

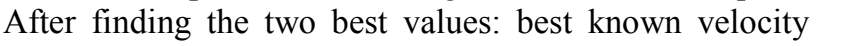

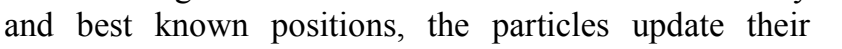

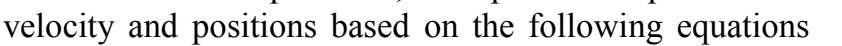
पाणाणाणा

$$
\begin{aligned}
& \nabla_{t}^{t+\square}=\chi \nabla_{t}^{t+\square}+C \square_{\square}^{t} \square t-x_{t}^{t} \square+C_{\square} \square^{t+\square} \square t-x_{t}^{t} \square \square \square \square
\end{aligned}
$$

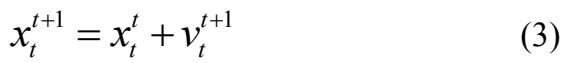

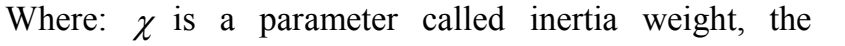

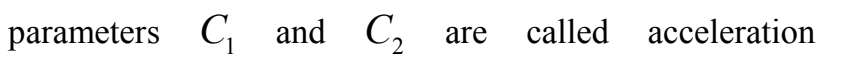

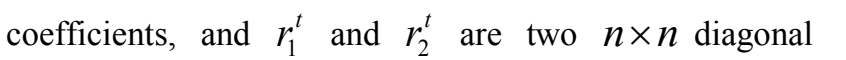

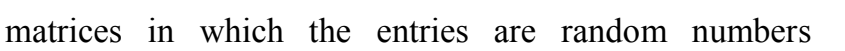

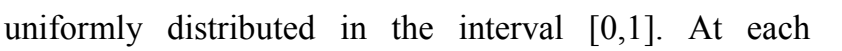

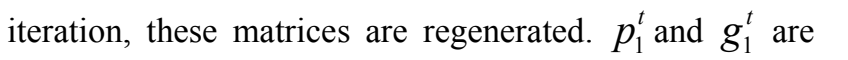

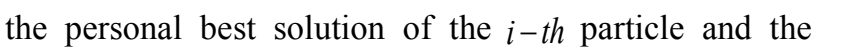

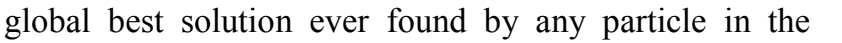

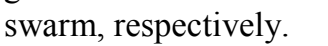

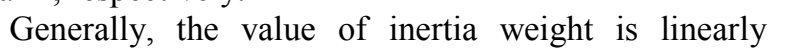

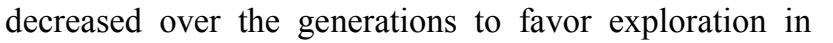

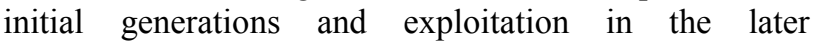

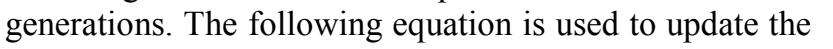

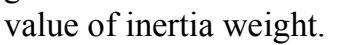

$$
\chi=\chi_{\square \square}-\frac{\chi_{\square \square}-\chi_{\square \square}}{\text { iter }_{\square \square}} \times \text { iter }
$$

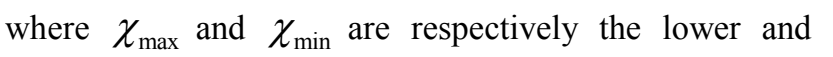

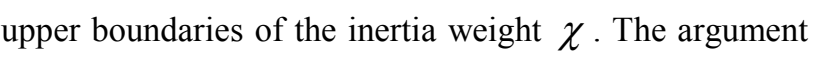

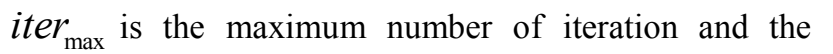

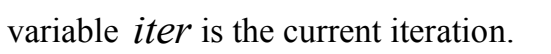

\subsection{Adaptive PSO-PID speed controller}

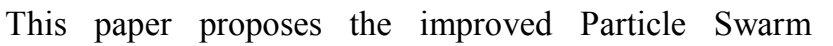

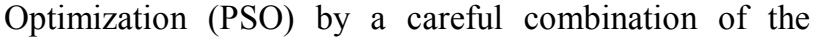

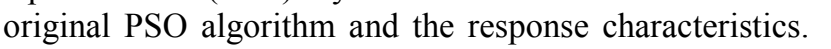

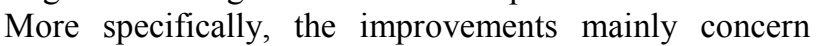

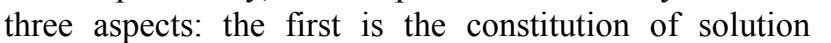

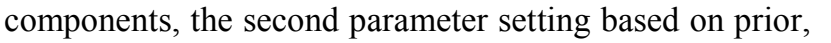

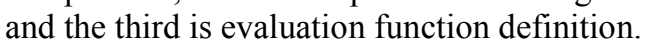

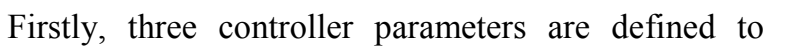

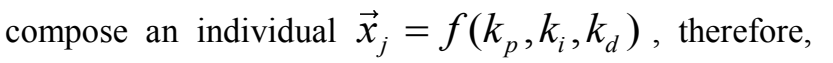

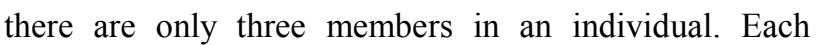

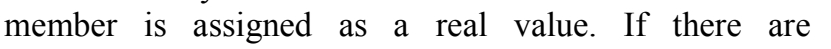

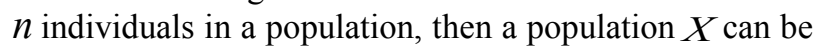

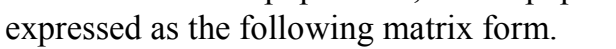

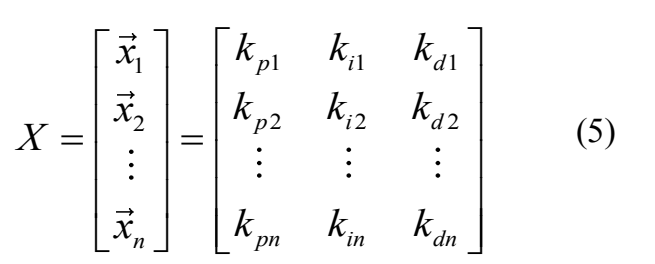

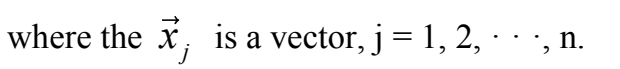

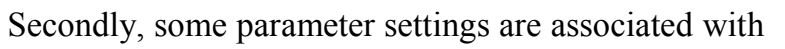

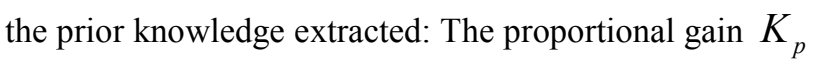

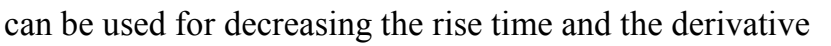

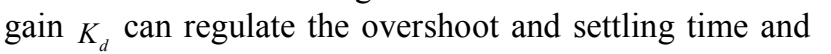

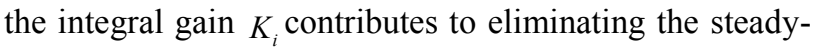

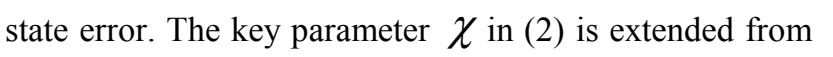

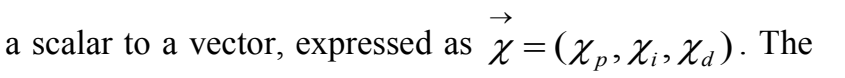

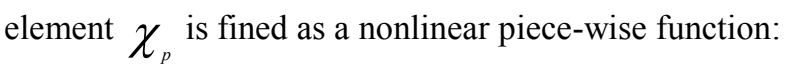




$$
\vec{\chi}_{p}=\left\{\begin{array}{c}
\chi_{\square} \text { if } \mid \delta-\phi \geq \sigma o r\left[e_{s s}\right] \geq \tau \\
\frac{\square}{\lambda}\left[\left(\chi_{\square}-\chi_{\square}\right)\left(\delta-\square+\chi_{\square}\right]\right.
\end{array}\right.
$$

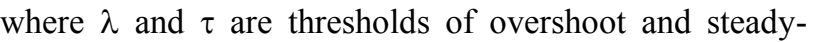

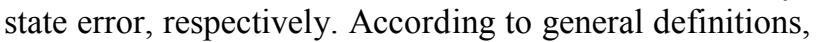

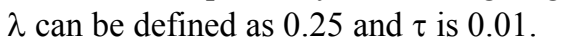

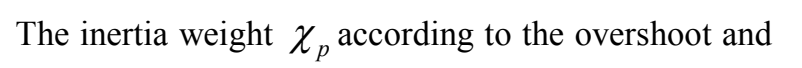
पणा।

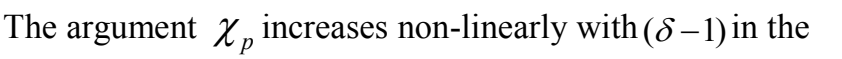

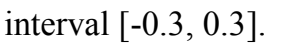

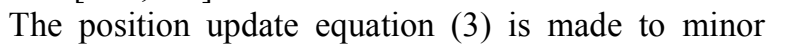

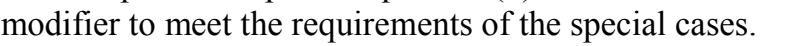

$$
x_{j \bar{p}}^{t+\square}=\left\{\begin{array}{l}
x_{j \bar{p}}^{t+\square}-\left|v_{j \bar{p}}^{t+\square}\right| \boldsymbol{e}_{s s} \geq \square \square \\
x_{j \bar{p}}^{t+\square}+\left|v_{j \bar{p}}^{t+\square}\right| \boldsymbol{e}_{s s} \leq-\square \square
\end{array}\right.
$$

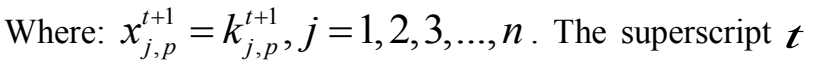
एणीण

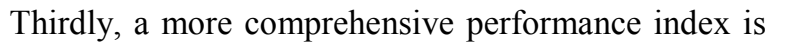

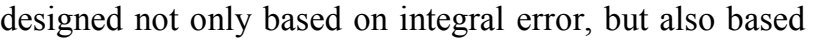
on the control input, rise time, settling time, etc... $\square$

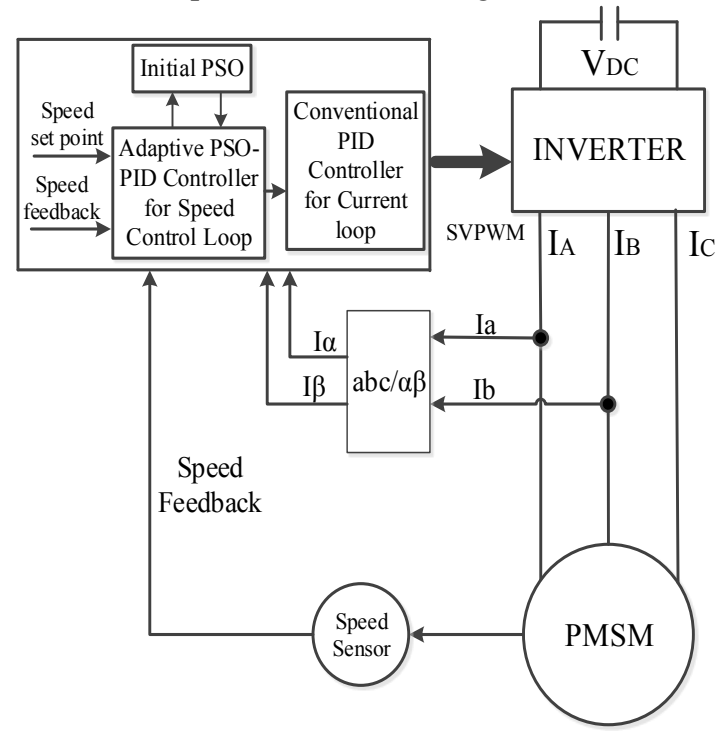

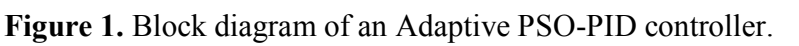
$\square$

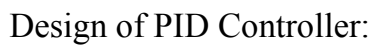

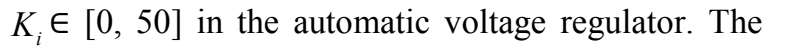

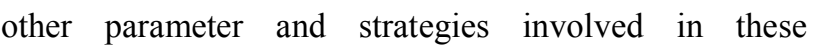
एवा

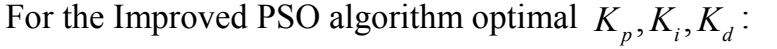

• $\quad \square\left[\begin{array}{ll}\text { - } \\ \text { • }\end{array}\right.$

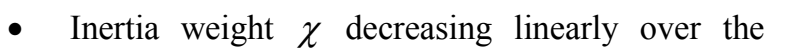

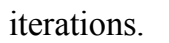

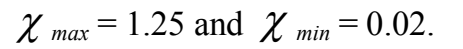

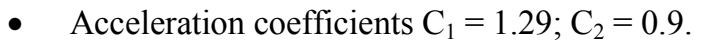

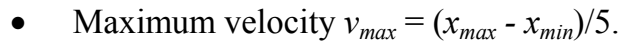

\begin{tabular}{|c|c|c|}
\hline \multicolumn{3}{|c|}{ PMSM Parameters } \\
\hline$\square \square \square\|ा\| ा \|$ & 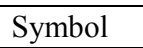 & $\square \square \| \square$ \\
\hline 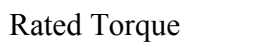 & $\square_{\square} \square$ & 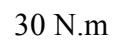 \\
\hline 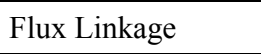 & $\psi_{f \square}$ & 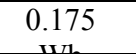 \\
\hline 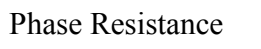 & $\square \square$ & $\square \| \mid \Omega \square$ \\
\hline 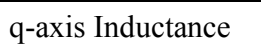 & $\square_{\square} \square$ & 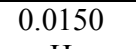 \\
\hline 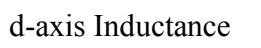 & $\square_{\square} \square$ & 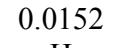 \\
\hline
\end{tabular}

Table 1. $\square\|\| \square \square\|m\| m \| \square \square \square \square$

$\square \square \square \square \square$

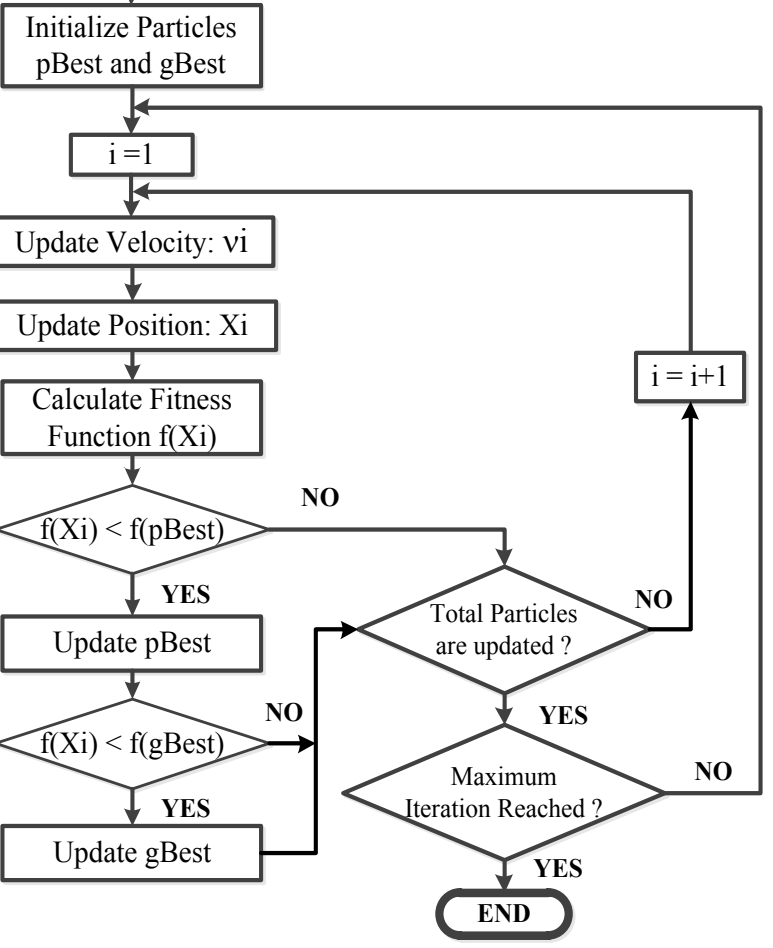

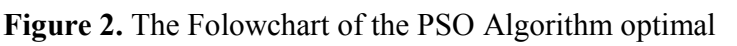

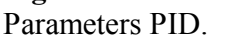

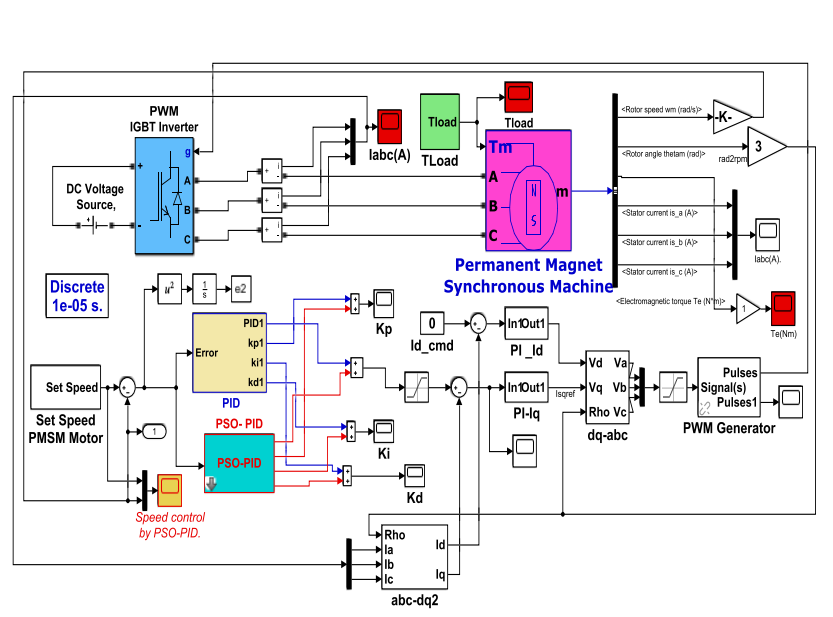

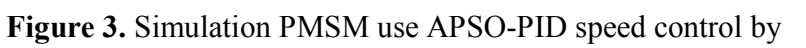

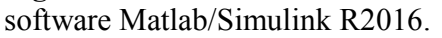




\section{Adaptive fuzzy-PID speed controller PMSM}

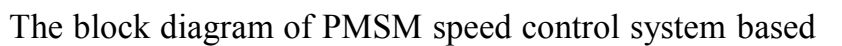

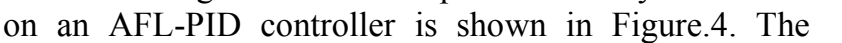

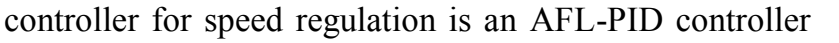

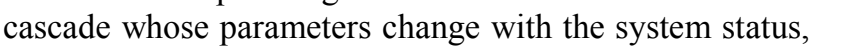

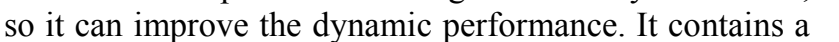

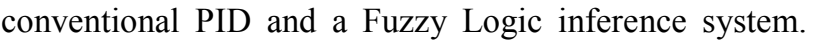

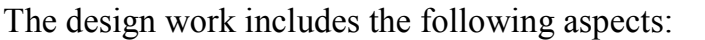

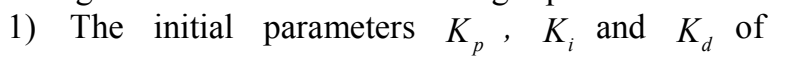

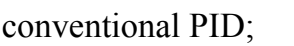

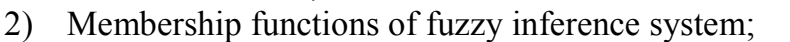

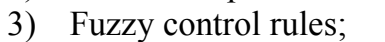

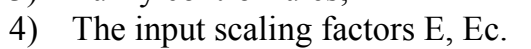

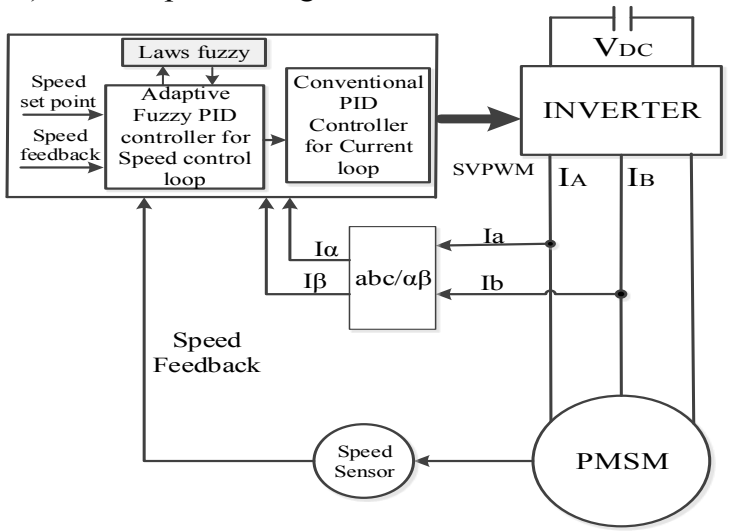

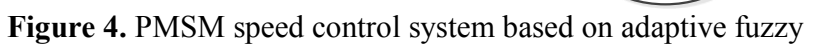
밈

\subsection{Design $K_{p}\left[K_{i} K_{d}\right.$ of an AFC-PID controller}

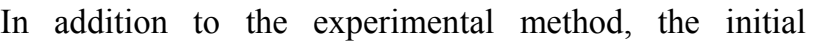

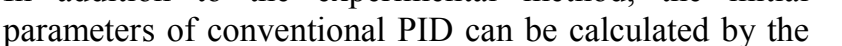

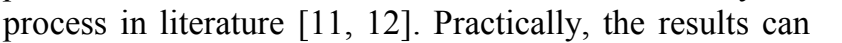

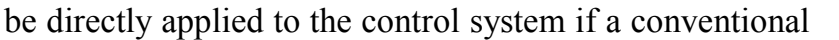

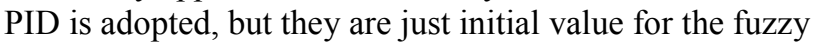

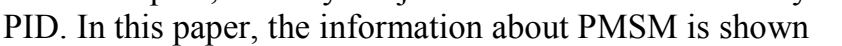

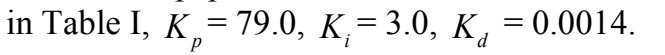

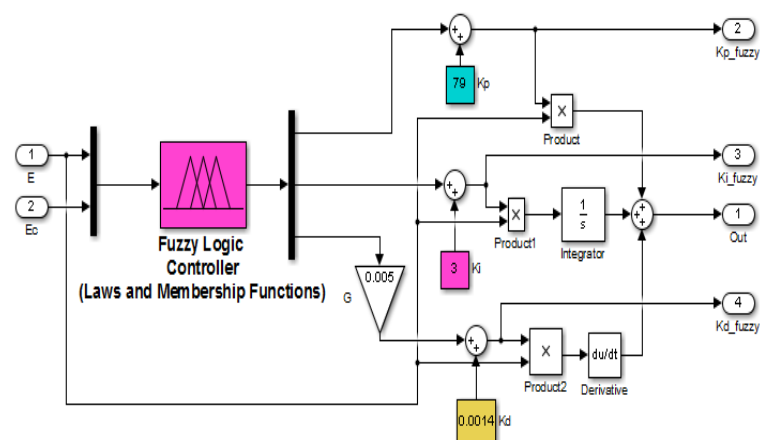

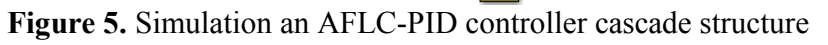

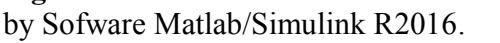

\subsection{Design of membership functions}

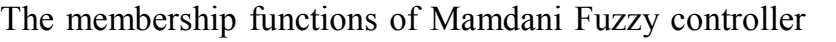

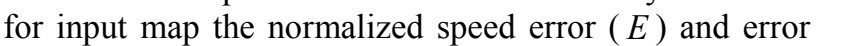

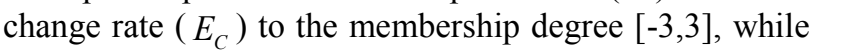

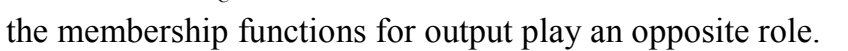

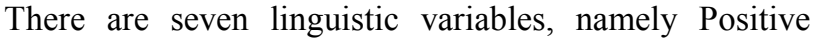

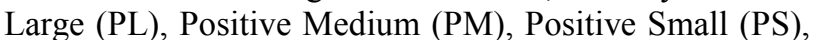

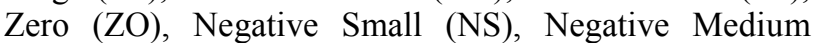

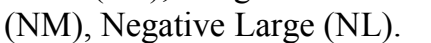
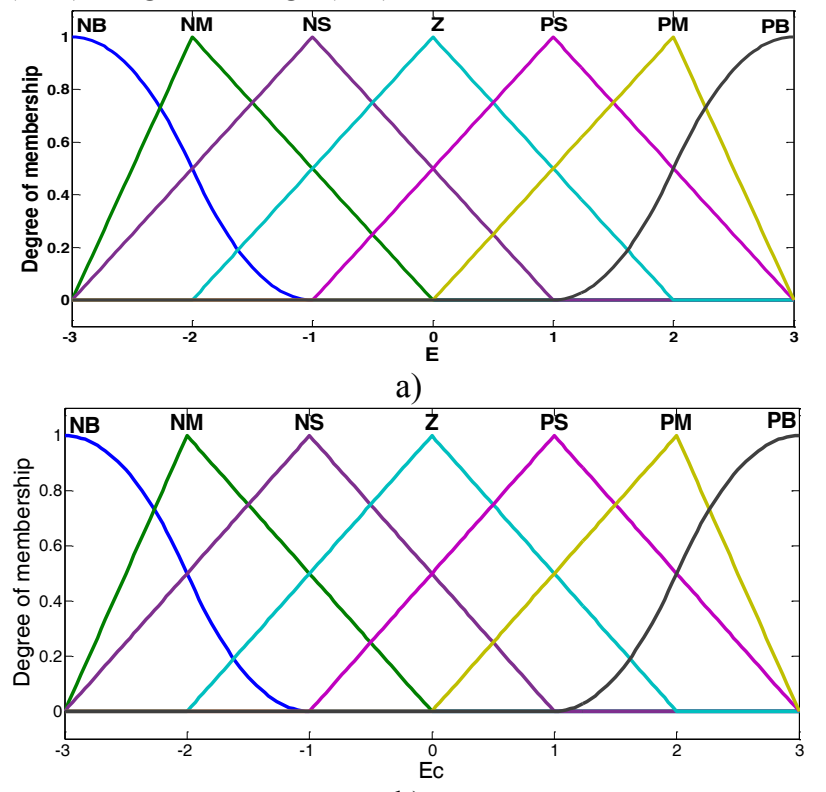

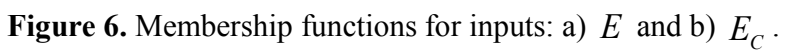

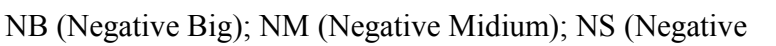

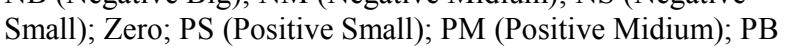

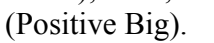
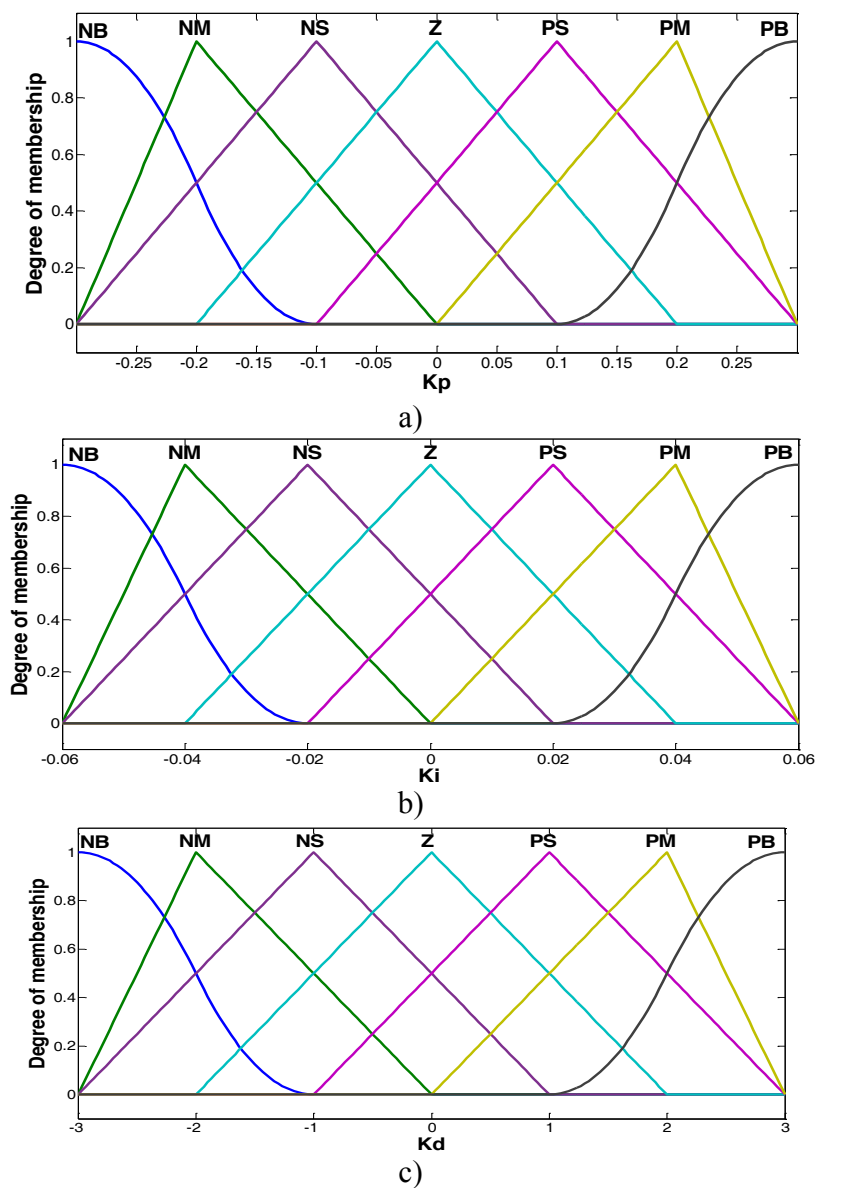

Figure 7. Membership $\square \square \square$ of outputs: a) $K_{p}$; b) $K_{i}$, c) $K_{d} \cdot \square$ 


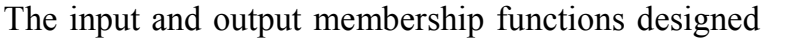
एण

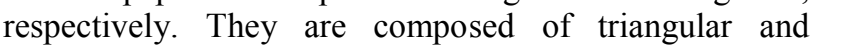

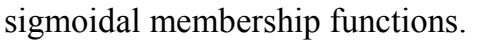

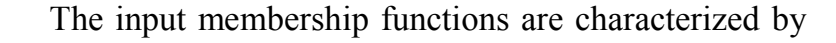
एव ए।

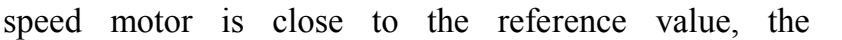

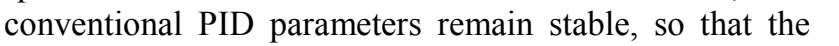

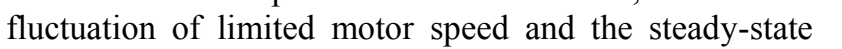

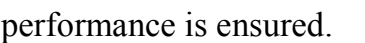

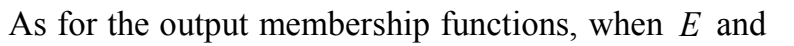

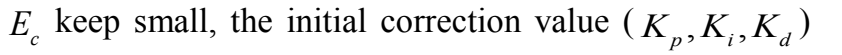

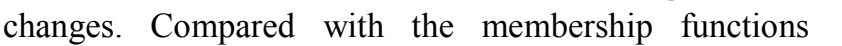

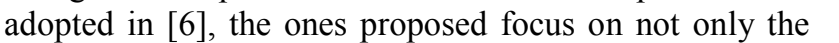

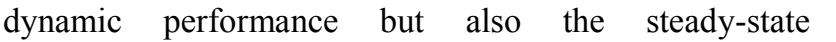

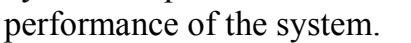

\subsection{Design of fuzzy control rules}

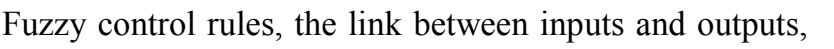

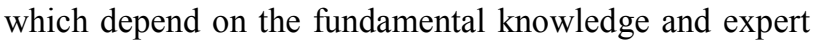

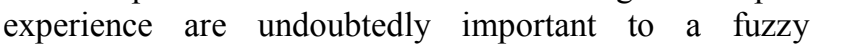

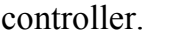

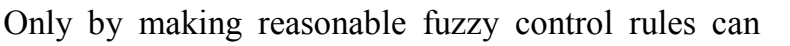
$\square \square \square$

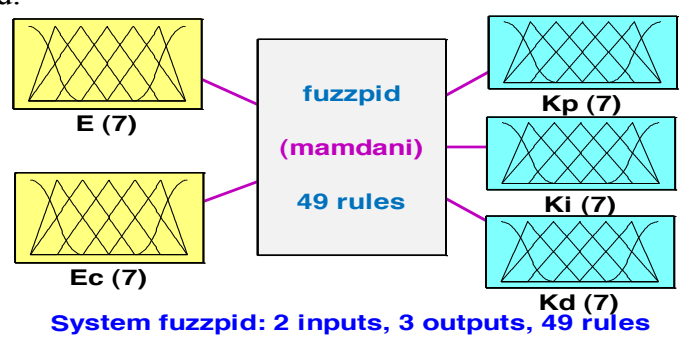

Figure 8. $\square \square \| K_{p}, K_{i} K_{d} \square \square \square \square$

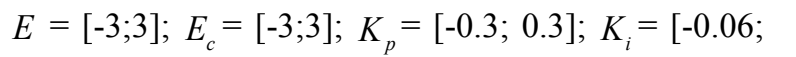

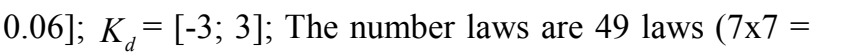
$\square \square \square \square \square ण \square$

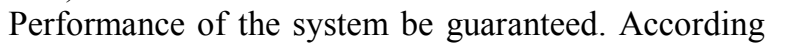

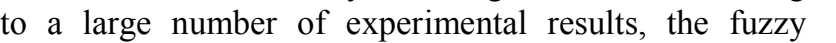

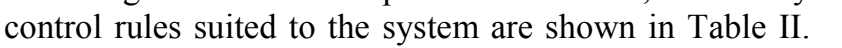

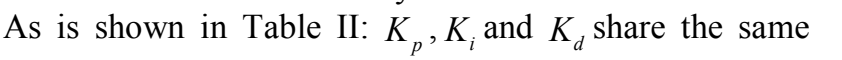

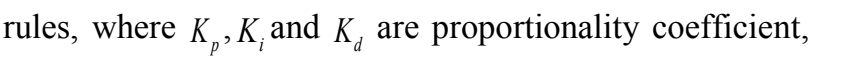

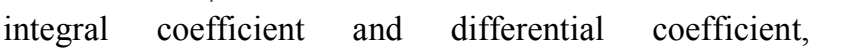

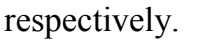

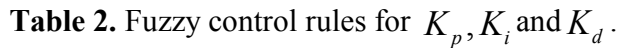

\begin{tabular}{|l|c|c|c|c|c|c|c|}
\hline EC & NB & NM & NS & Z & PS & PM & PB \\
\hline NB & $\square \square \square$ & $\square \square \square$ & $\square \square$ & $\square \square$ & $\square \square$ & $\square \square$ & $\square \square \square$ \\
\hline NM & $\square \square \square$ & $\square \square \square$ & $\square \square$ & $\square \square$ & $\square \square$ & $\square \square$ & $\square \square \square$ \\
\hline NS & $\square \square \square$ & $\square \square \square$ & $\square \square \square$ & $\square \square$ & $\square \square$ & $\square \square$ & $\square \square$ \\
\hline Z & $\square \square \square$ & $\square \square \square$ & $\square \square \square$ & $\square \square$ & $\square \square \square$ & $\square \square$ & $\square \square \square$ \\
\hline PS & $\square \square \square$ & $\square \square \square$ & $\square \square \square$ & $\square \square$ & $\square \square$ & $\square \square$ & $\square \square \square$ \\
\hline PM & $\square \square \square$ & $\square \square \square$ & $\square \square$ & $\square \square$ & $\square \square$ & $\square \square$ & $\square \square \square$ \\
\hline PB & $\square \square \square$ & $\square \square \square$ & $\square \square \square$ & $\square \square$ & $\square \square \square$ & $\square \square$ & $\square \square \square$ \\
\hline
\end{tabular}

\subsection{Design of output scale factors}

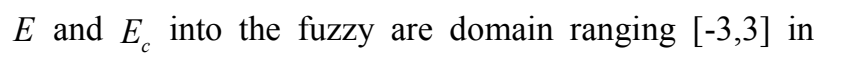

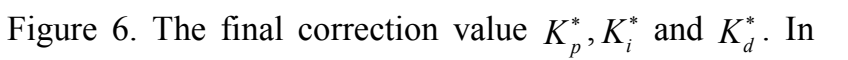

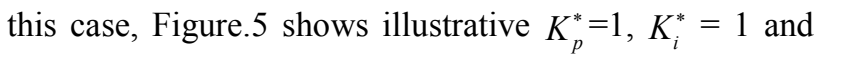
$K_{d}^{*}$ पाणा|ण

\subsection{AFL-PID controller speed PMSM Motor}

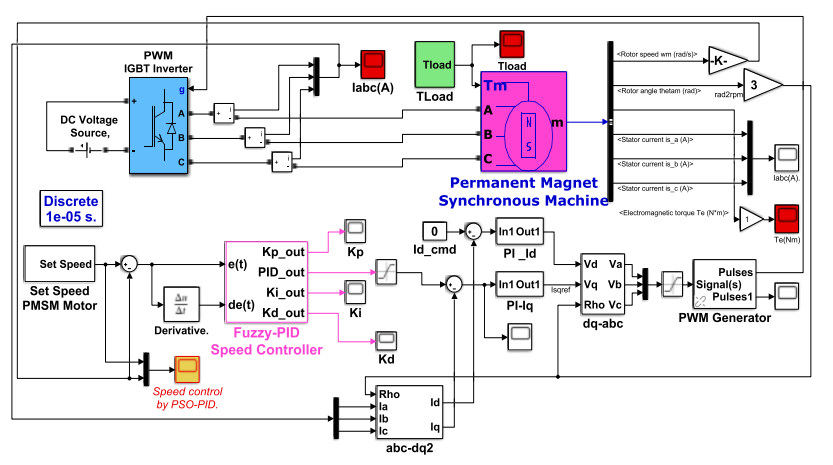

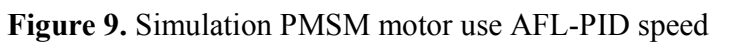

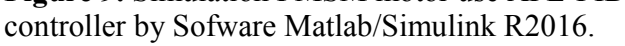

\section{Simulation results}

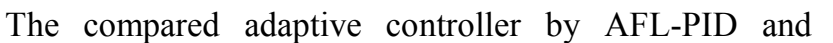

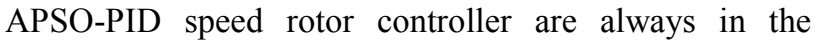

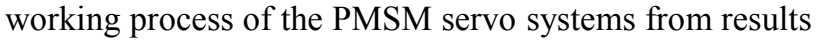

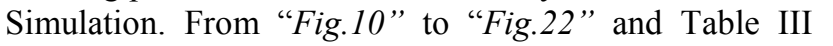

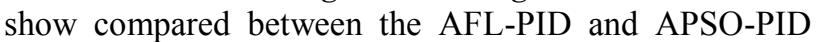

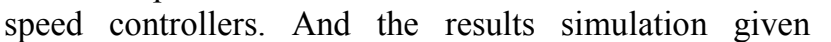

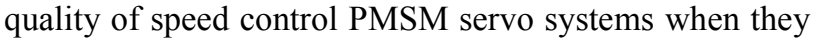

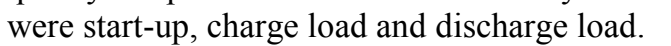

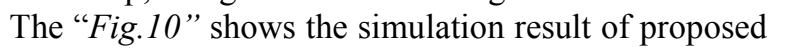

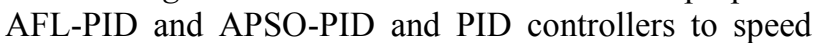

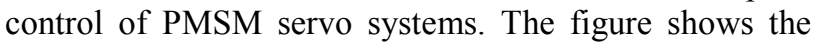

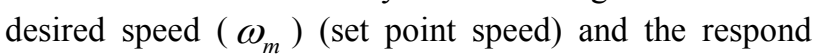

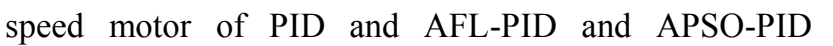
पा

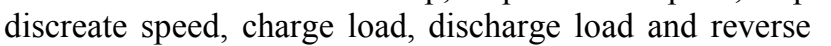

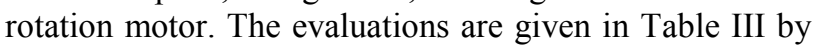
the numbers and images in "Fig.11" to "Fig.22" $\square$

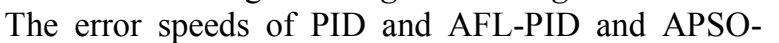

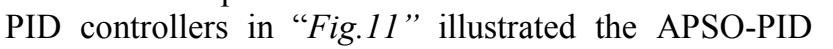

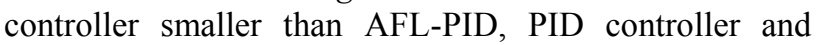

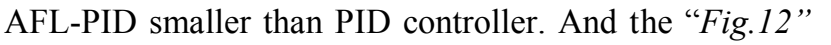

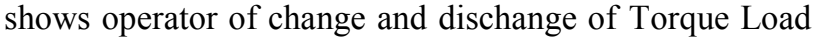

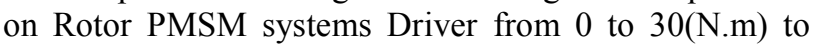

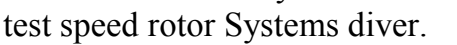

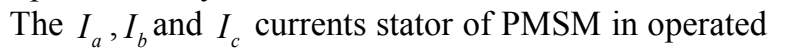

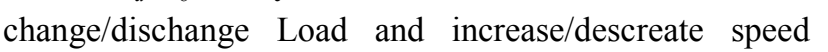

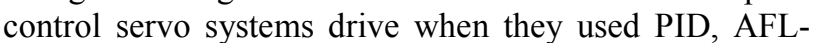

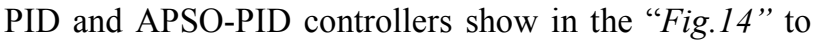

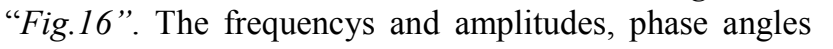

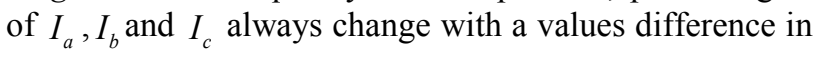

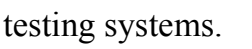




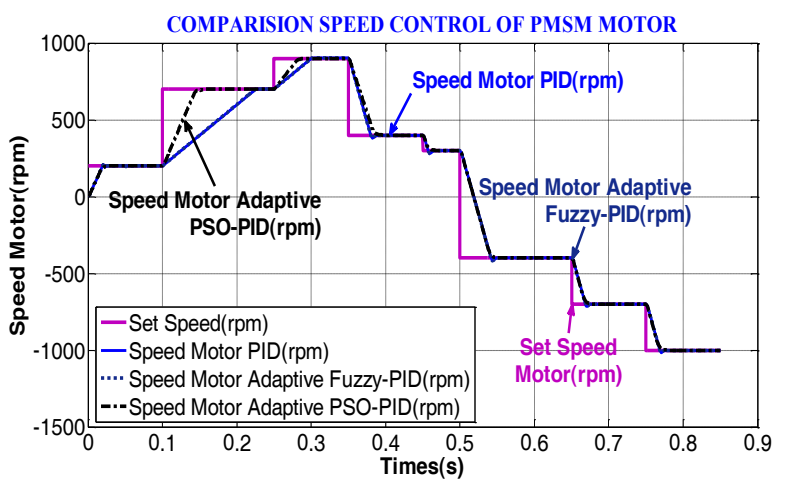

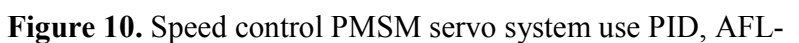

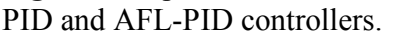

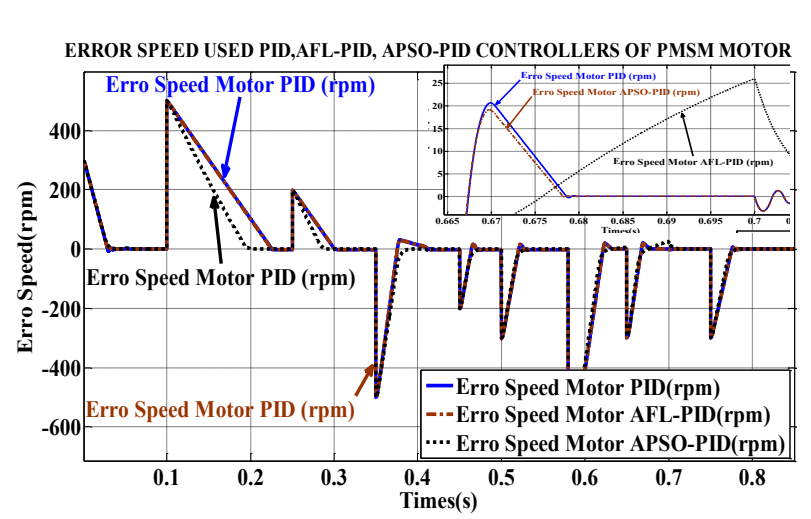

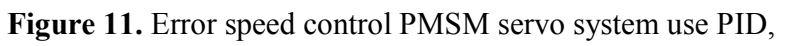

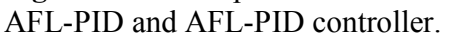

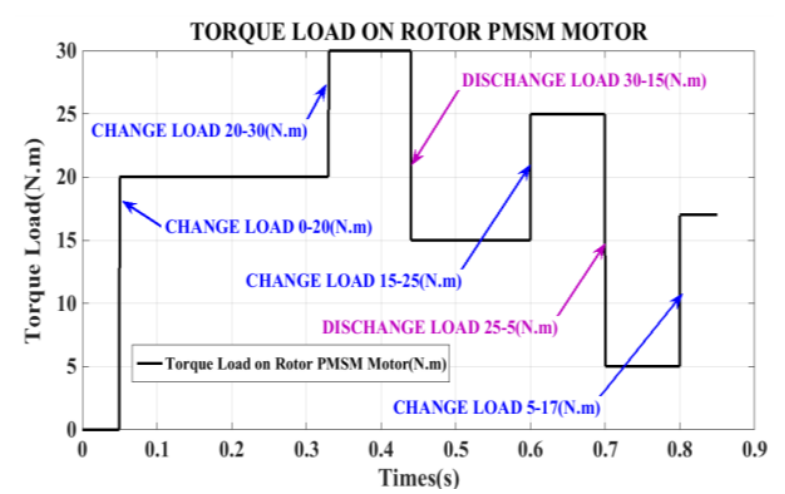

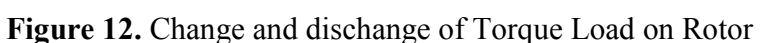

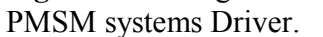

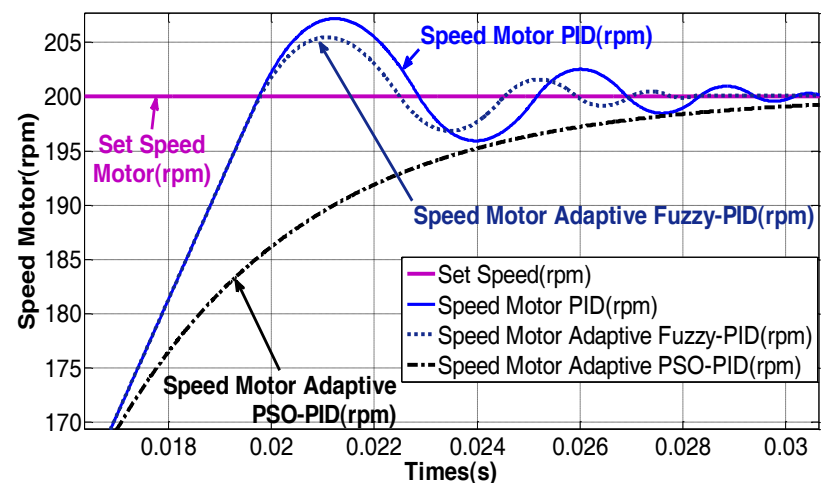

Figure 13. $\square \square \square \square m$ m

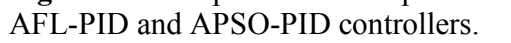

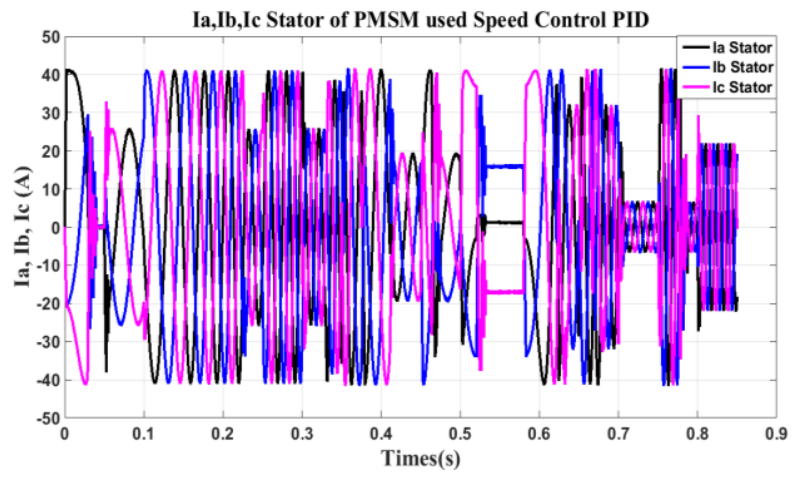

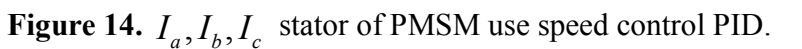

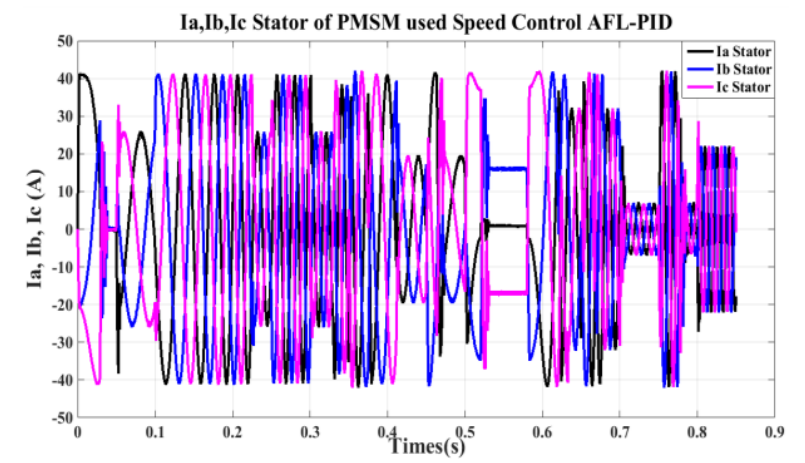

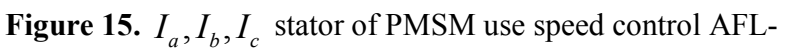
$\square \square \square$

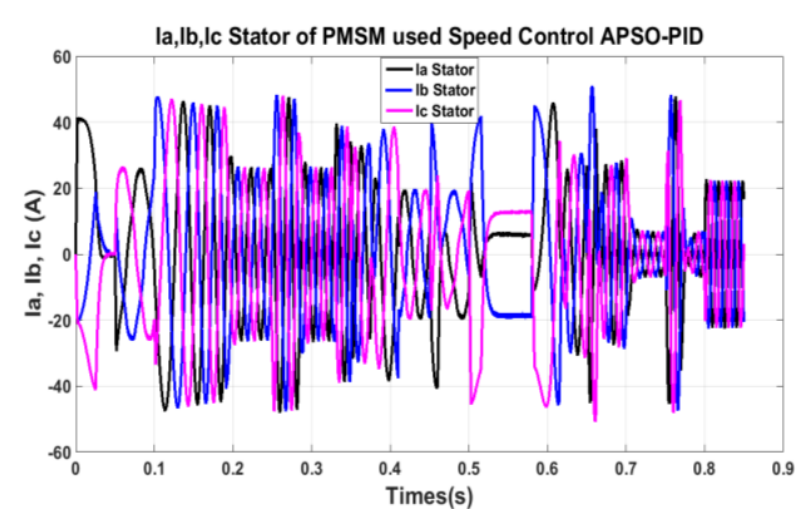

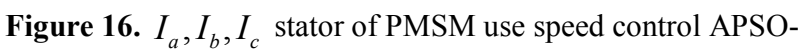

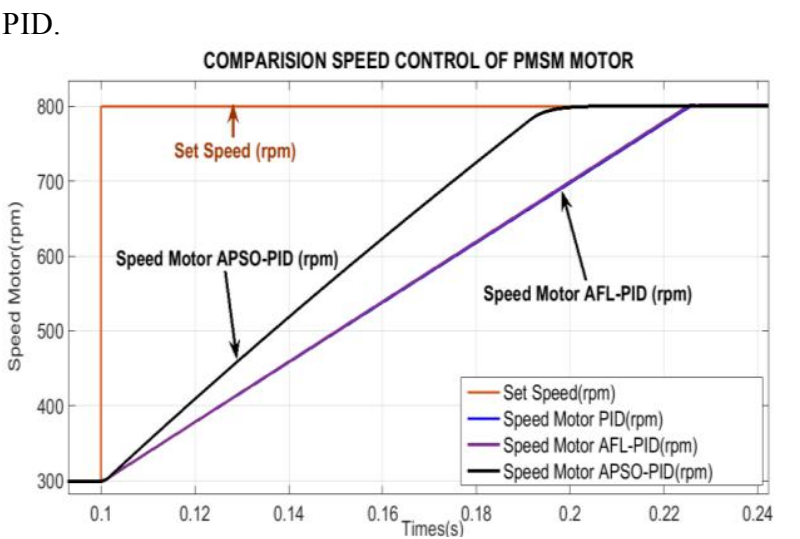

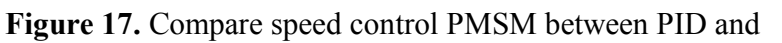

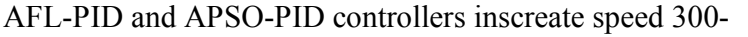
$\square 1\|\| \square \square \square$ 


\section{COMPARISION SPEED CONTROL OF PMSM MOTOR}

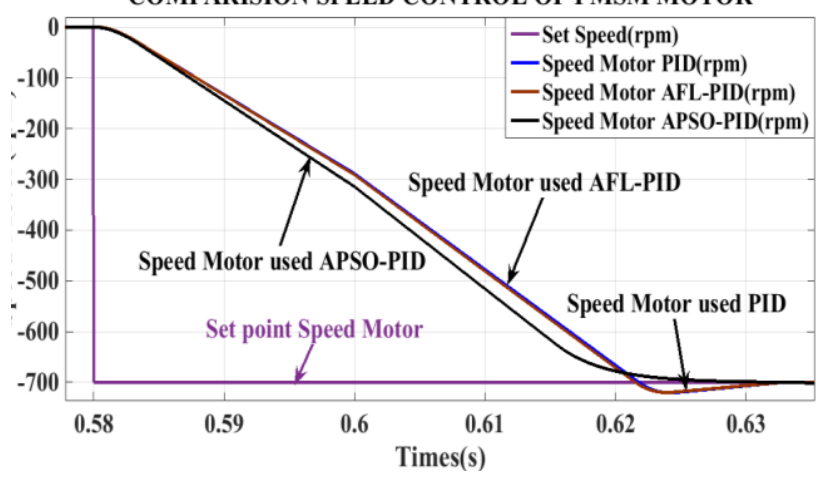

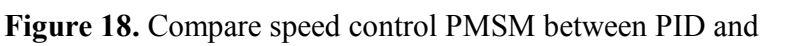

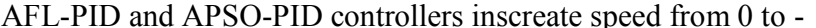
$\square 11 \mathrm{~m} \square \square \square$

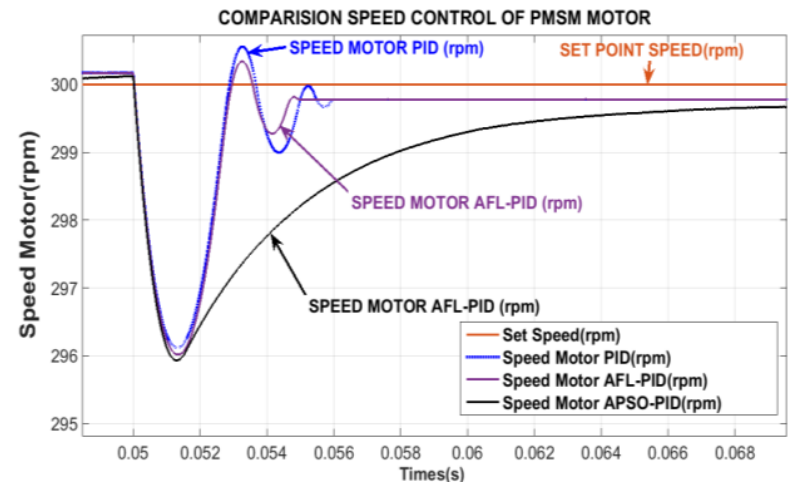

Figure 19. $\square \square \square \square \|$ m

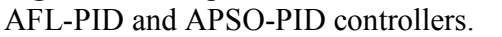

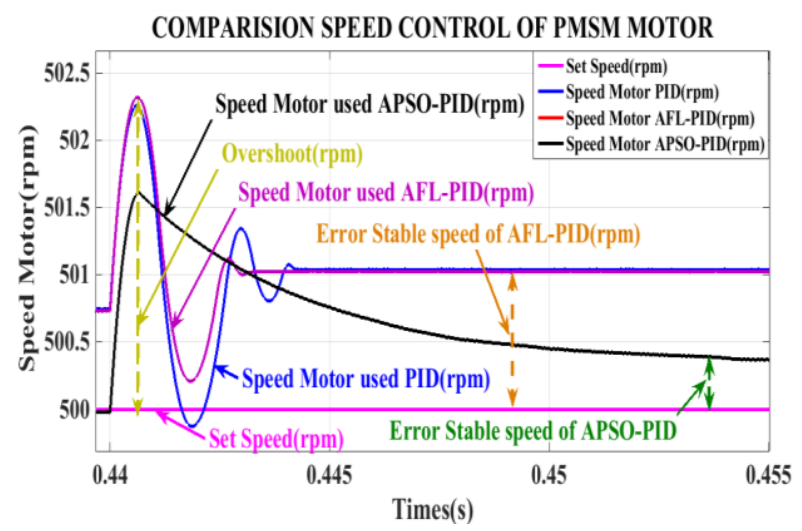

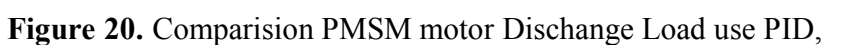

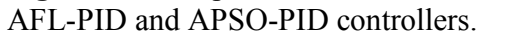

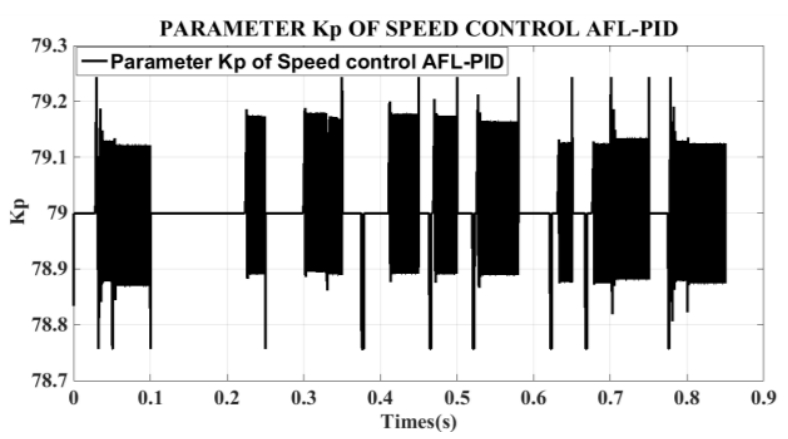

$\square$

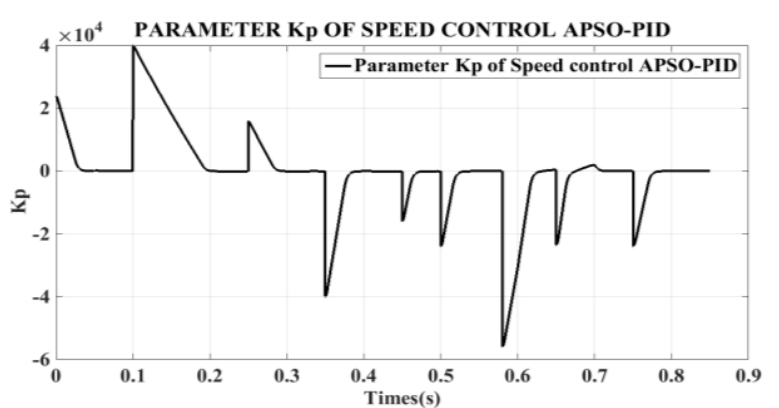

$\square$

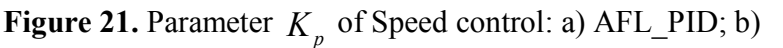

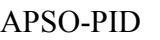

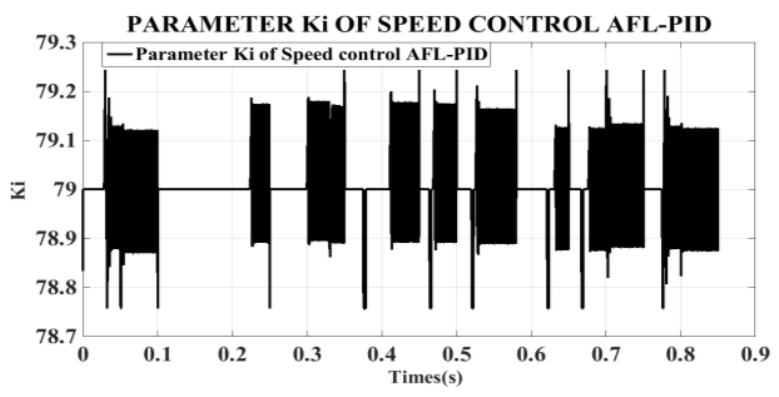

$\square \square$

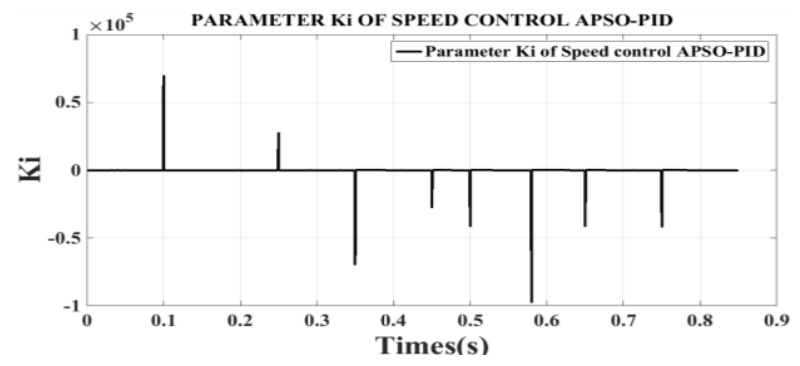

$\square$

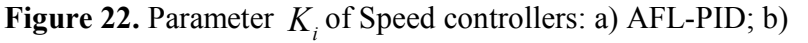

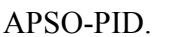
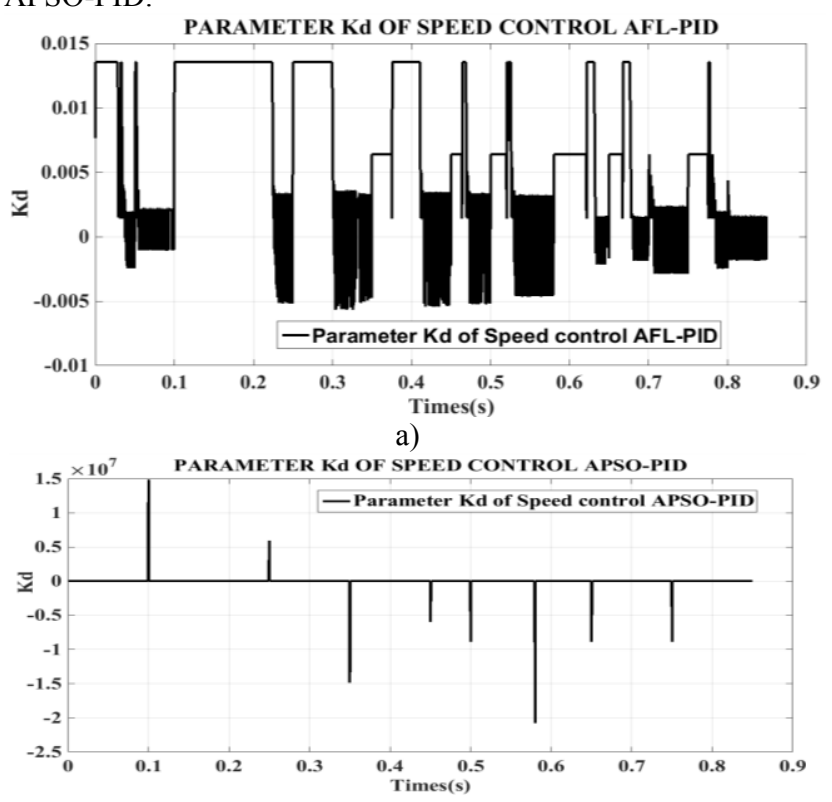

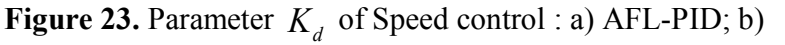




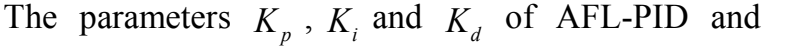

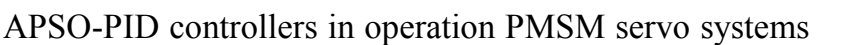

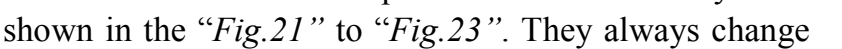

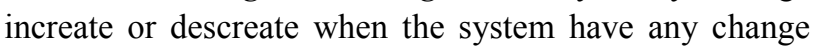

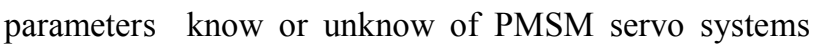

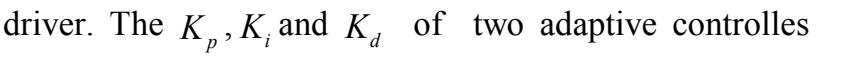

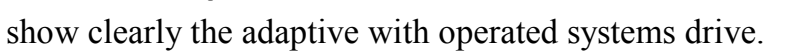

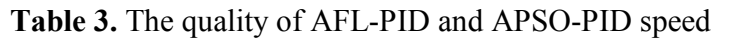

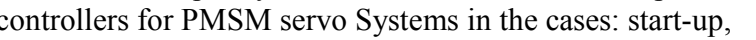

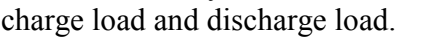

\begin{tabular}{|c|c|c|c|c|c|c|}
\hline \multirow{2}{*}{$\begin{array}{l}\text { Controller } \\
\end{array}$} & \multicolumn{2}{|c|}{$\begin{array}{c}\text { Start-up } \\
0-200(\text { rpm) }\end{array}$} & \multicolumn{2}{|c|}{$\begin{array}{c}\text { Discharge load } T_{L} \\
\text { from 30N.m to } 15 \\
\text { N.m at } t=0.44(s)\end{array}$} & \multicolumn{2}{|c|}{$\begin{array}{c}\text { Charge load } T_{L} \\
\text { from } 0 \text { N.m to } \\
20 N . m \text { at } \\
t=0.05(s)\end{array}$} \\
\hline & AFL-PID & $\begin{array}{c}\text { APSO- } \\
\text { PID }\end{array}$ & $\begin{array}{c}A F L- \\
P I D\end{array}$ & $\begin{array}{c}\text { APSO- } \\
\text { PID }\end{array}$ & $\begin{array}{c}A F L- \\
P I D\end{array}$ & $\begin{array}{c}\text { APSO- } \\
\text { PID }\end{array}$ \\
\hline $\begin{array}{c}\Delta \omega_{M \square} \\
(r p m)\end{array}$ & $\square ण$ & $\square$ & पाणा & $\square ण$ & पा山ा & $\square$ \\
\hline 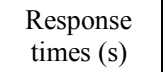 & पणा & पणा & पाणा & पणाए & एणाए & पणा। \\
\hline 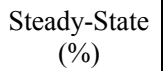 & $\square ण$ & पाण & पाण & पाण & एणाए & पणा। \\
\hline 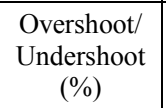 & पा山 & पाण & पणा & पाण & पणाए & पाण \\
\hline 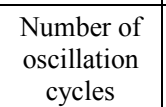 & $\square$ & $\square$ & $\square$ & $\square$ & ए & $\square$ \\
\hline
\end{tabular}

\section{Simulation results}

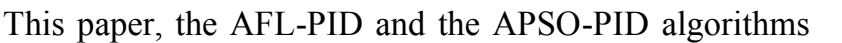

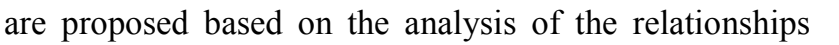

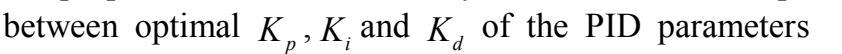

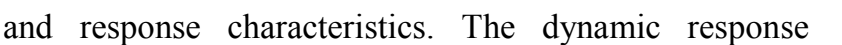

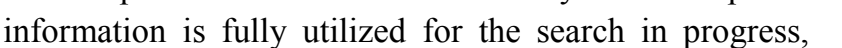

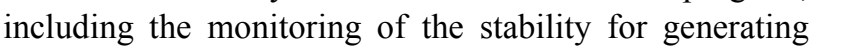

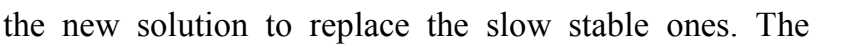

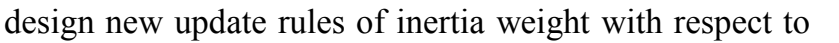

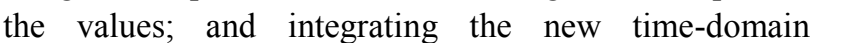

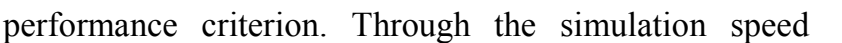

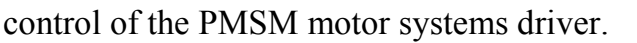

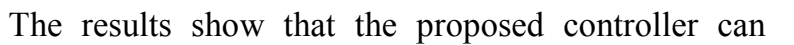

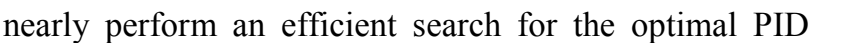

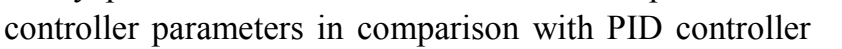

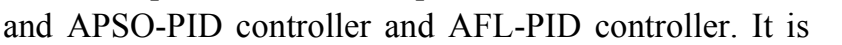

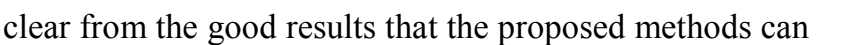

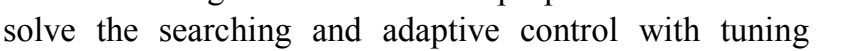

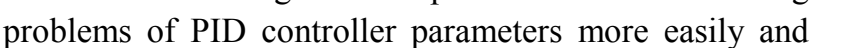

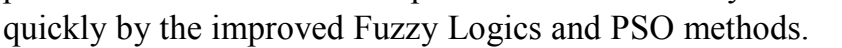

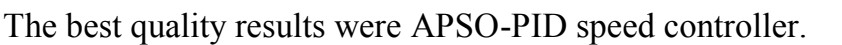

\section{References}

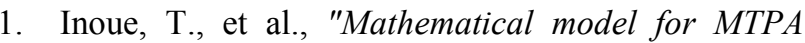
control of permanent-magnet synchronous motor in stator flux linkage synchronous frame," $\square \square \square \square \square$

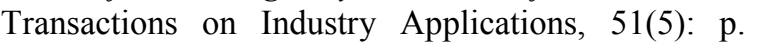

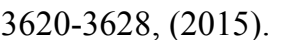

$\square \square \square \square\|\square \square\| \square \square \| ा \square \square \square \square \square \square \square$ 'Predictive speed control with short prediction horizon for permanent magnet synchronous motor drives, $\quad \square \quad \square \square \square \square$

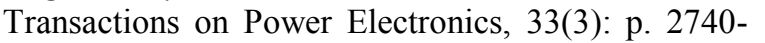

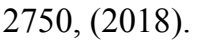

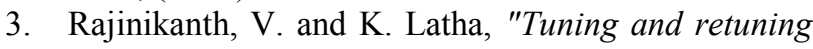
of PID controller for unstable systems using

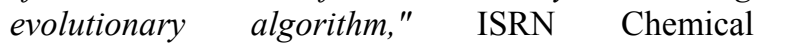

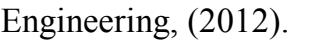

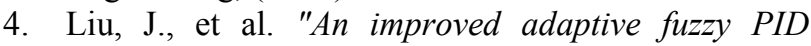
controller for PMSM and a novel stability analysis

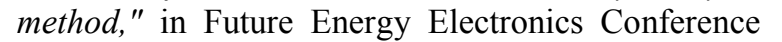

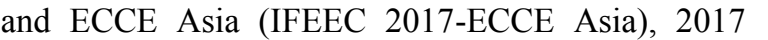

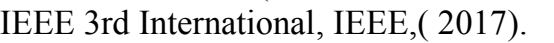

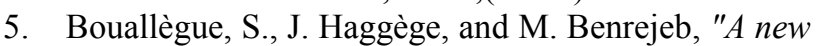
method for tuning PID-type fuzzy controllers using

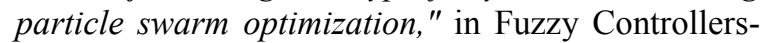

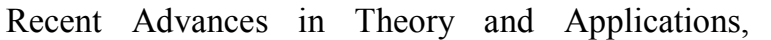

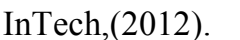

$\square \quad \square \square \| \square \square \square \square \square \square \square \square$ "A Particle Swarm Optimization-PID controller of a DC Servomotor for

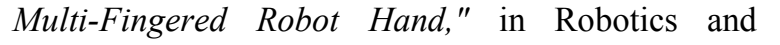

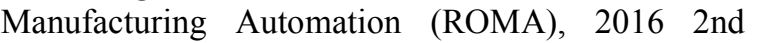

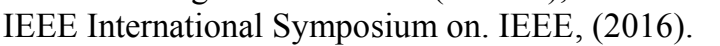

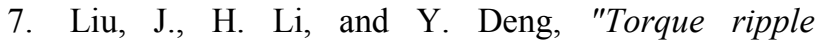
minimization of pmsm based on robust ilc via

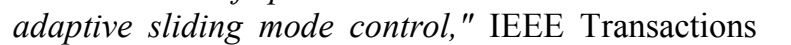

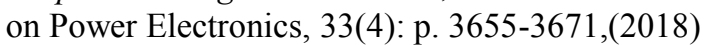

$\square \quad \square \square\|\| \square \square \square \square \square \square$ "Self-organizing hierarchical particle swarm optimization of correlation filters for

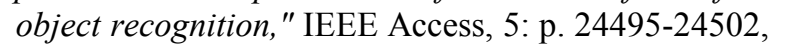

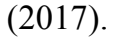

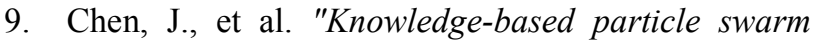
optimization for PID controller tuning," $\square \square$

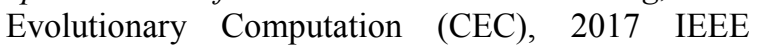

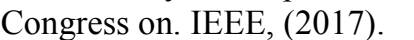

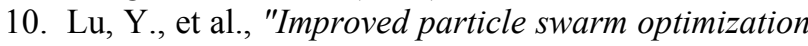
algorithm and its application in text feature

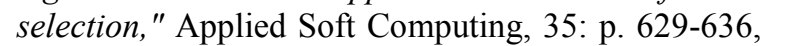

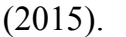

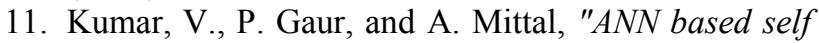
tuned PID like adaptive controller design for high performance PMSM position control," $\square \square \square \square \square \square$

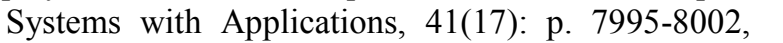
एमामाप

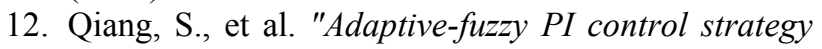
for flux- $\square \square \square \square$ permanent magnet motors, $" \square \square$

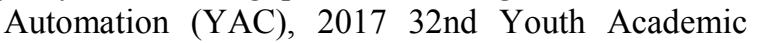

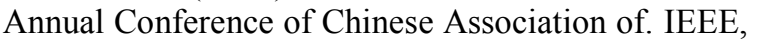
एणा1ा 